\title{
Comparison of prostatic artery embolisation (PAE) versus transurethral resection of the prostate (TURP) for benign prostatic hyperplasia: randomised, open label, non-inferiority trial
}

${ }^{1}$ Department of Urology, St

Gallen Cantonal Hospital, 9007

St Gallen, Switzerland

${ }^{2}$ Department of Radiology and Nuclear Medicine, St Gallen Cantonal Hospital, St Gallen, Switzerland

${ }^{3}$ Clinical Trials Unit, St Gallen Cantonal Hospital, St Gallen, Switzerland

${ }^{4}$ Neuro-Urology, Spinal Cord Injury Center and Research, University of Zürich, Balgrist University Hospital, Zürich, Switzerland

Correspondence to:

D Abt dominik.abt@kssg.ch (or @AbtDominik on Twitter)

Additional material is published online only. To view please visit the journal online.

Cite this as: $B M J$ 2018;361:k2338 http://dx.doi.org/10.1136/bmj.k2338

Accepted: 6 May 2018
Dominik Abt, ${ }^{1}$ Lukas Hechelhammer, ${ }^{2}$ Gautier Müllhaupt, ${ }^{1}$ Stefan Markart, ${ }^{2}$ Sabine Güsewell, ${ }^{3}$ Thomas M Kessler, ${ }^{4}$ Hans-Peter Schmid, ${ }^{1}$ Daniel S Engeler, ${ }^{1}$ Livio Mordasini ${ }^{1}$

\begin{abstract}
Objective

To compare prostatic artery embolisation (PAE) with transurethral resection of the prostate (TURP) in the treatment of lower urinary tract symptoms secondary to benign prostatic hyperplasia in terms of patient reported and functional outcomes.

Design

Randomised, open label, non-inferiority trial.

Setting

Urology and radiology departments of a Swiss tertiary care centre.
\end{abstract}

Participants

103 patients aged $\geq 40$ years with refractory lower urinary tract symptoms secondary to benign prostatic hyperplasia were randomised between 11 February 2014 and 24 May 2017; 48 and 51 patients reached the primary endpoint 12 weeks after PAE and TURP, respectively.

Interventions

PAE performed with 250-400 $\mu$ m microspheres under local anaesthesia versus monopolar TURP performed under spinal or general anaesthesia.

Main outcomes and measures

Primary outcome was change in international prostate symptoms score (IPSS) from baseline to 12 weeks after surgery; a difference of less than 3 points

\section{WHAT IS ALREADY KNOWN ON THIS TOPIC}

Prostatic artery embolisation (PAE) for the treatment of benign prostatic

hyperplasia has been introduced into clinical practice without high level

evidence, and is now increasingly performed worldwide

Available PAE data point to promising outcomes, including a favourable safety profile

Only three trials so far have included a control group receiving established surgical treatments for benign prostatic hyperplasia; all studies performed so far have been criticised for methodological drawbacks and have shown highly contradictory results

\section{WHAT THIS STUDY ADDS}

An improvement in symptoms close to that achieved by transurethral resection of the prostate (TURP) was found after PAE in the short term

An inferior desobstructive effect and a favourable side effect profile were found for PAE, and should be considered for patient selection

Further comparative study findings (including longer follow-up periods) should be evaluated before PAE can be considered as a routine treatment between treatments was defined as non-inferiority for PAE and tested with a one sided $t$ test. Secondary outcomes included further questionnaires, functional measures, magnetic resonance imaging findings, and adverse events; changes from baseline to 12 weeks were compared between treatments with two sided tests for superiority.

Results

Mean reduction in IPSS from baseline to 12 weeks was -9.23 points after PAE and -10.77 points after TURP. Although the difference was less than 3 points (1.54 points in favour of TURP ( $95 \%$ confidence interval -1.45 to 4.52$)$ ), non-inferiority of PAE could not be shown $(P=0.17)$. None of the patient reported secondary outcomes differed significantly between treatments when tested for superiority; IPSS also did not differ significantly $(P=0.31)$. At 12 weeks, $P A E$ was less effective than TURP regarding changes in maximum rate of urinary flow $(5.19 v 15.34 \mathrm{~mL} / \mathrm{s}$; difference 10.15 (95\% confidence interval -14.67 to $-5.63) ; \mathrm{P}<0.001)$, postvoid residual urine $(-86.36 \mathrm{~V}$ $-199.98 \mathrm{~mL} ; 113.62$ (39.25 to 187.98); $\mathrm{P}=0.003)$, prostate volume $(-12.17 \mathrm{v}-30.27 \mathrm{~mL} ; 18.11$ (10.11 to 26.10); P 0.001), and desobstructive effectiveness according to pressure flow studies (56\% v 93\% shift towards less obstructive category; $\mathrm{P}=0.003$ ). Fewer adverse events occurred after PAE than after TURP (36 $v 70$ events; $\mathrm{P}=0.003)$.

Conclusions

The improvement in lower urinary tract symptoms secondary to benign prostatic hyperplasia seen 12 weeks after PAE is close to that after TURP. PAE is associated with fewer complications than TURP but has disadvantages regarding functional outcomes, which should be considered when selecting patients. Further comparative study findings, including longer follow-up, should be evaluated before PAE can be considered as a routine treatment.

Trial registration

Clinicaltrials.gov NCT02054013.

\section{Introduction}

Benign prostatic hyperplasia is one of the most common diseases in men and is often associated with bladder outlet obstruction and lower urinary tract symptoms. The incidence of benign prostatic hyperplasia in men aged 50-60 years is 50\% and rises with increasing age. ${ }^{1}$ Treatment for the disease incurs a substantial economic burden, with estimated annual costs of 
up to US\$4 billion ( $€ 3.01 \mathrm{bn}$; €3.45bn) in the United States in 2006, and mean annual treatment costs of $€ 858$ per patient in Europe in 2003 . $^{23}$ Owing to the demographic shift towards an elderly population, costs arising from lower urinary tract symptoms secondary to benign prostatic hyperplasia (BPH-LUTS) are likely to increase substantially. Surgical treatment is recommended if conservative treatment fails or for patients with complications related to benign prostatic hyperplasia, and is performed in more than 100000 men annually in the USA. ${ }^{4}$ Transurethral resection of the prostate (TURP) is still the surgical gold standard in most patients. ${ }^{56}$ However, it is associated with high morbidity, ${ }^{78}$ and $40 \%$ of patients have residual lower urinary tract symptoms that require drug treatment within five years after surgery. ${ }^{9}$ These drawbacks have led to a continuous search for less invasive treatments.

Improvements in BPH-LUTS after prostatic artery embolisation (PAE) - a minimally invasive procedure that can be performed under local anaesthesia-were first reported in $2000 .^{10}$ Since then, nearly 1000 cases have been published, showing significant improvements in BPH-LUTS, a favourable side effect profile, and lower treatment costs than with TURP. ${ }^{11}$

12 Considering these advantages, non-inferiority of PAE compared with TURP as a reference treatment would support its implementation as a treatment for BPH-LUTS. However, all studies performed so far have been criticised for methodological drawbacks; only three trials have included a control group that received established surgical treatments for benign prostatic hyperplasia, and the results were highly contradictory. ${ }^{13-15}$

The discussion regarding the use of PAE to treat BPH-LUTS is highly controversial and is influenced by political interests, as a fundamental domain of urologists is challenged by interventional radiologists. Evidence to support the use of PAE in the treatment of BPH-LUTS is so far insufficient, and PAE is still considered experimental. ${ }^{1116}$ The lack of high quality evidence comparing PAE and TURP is a crucial knowledge gap in urology. We therefore compared the efficacy and safety of the two interventions in a randomised, open label, non-inferiority trial.

\section{Methods}

Trial design

This investigator initiated, open label, single centre, randomised controlled trial was performed with the close collaboration of the departments of urology and radiology at St Gallen Cantonal Hospital, Switzerland. The trial was designed by the lead investigators and supported by biostatisticians from the hospital's clinical trials unit. The study protocol was reviewed by experts from the clinical trials unit and approved by the local ethics committee (EKSG14/004). The study was funded by a grant from the hospital's research committee (14/08), and had no commercial support. Independent experts from the clinical trials unit performed study coordination, data management, and data and safety monitoring.
SecuTrial (Interactive Systems) was used for electronic trial data management. The clinical trials unit's statistician (SG) performed the data analyses. Trained study physicians collected patient information and undertook enrolment and randomisation. The trial was performed according to the World Medical Association Declaration of Helsinki ${ }^{17}$ and the Guidelines for Good Clinical Practice. ${ }^{18}$ The study protocol was published ${ }^{19}$ and registered at ClinicalTrials.gov (NCT02054013).

\section{Participants}

The trial aimed to recruit a population with refractory BPH-LUTS that is typically treated with TURP in everyday clinical practice. Inclusion criteria were men aged at least 40 years, TURP indicated, refractory to medical treatment or not willing to undergo or continue medical treatment, with a prostate size 25-80 $\mathrm{mL}$ as measured by transabdominal ultrasound, with an international prostate symptoms score (IPSS) of at least 8, with an IPSS related quality of life of at least 3 points, with a maximum urinary flow rate of less than $12 \mathrm{~mL} / \mathrm{s}$ or urinary retention, and who provided written informed consent.

Exclusion criteria were severe atherosclerosis, aneurysmatic changes or severe tortuosity in the aortic bifurcation or internal iliac arteries, acontractile detrusor, neurogenic lower urinary tract dysfunction, urethral stenosis, bladder diverticulum, bladder stone, allergy to intravenous contrast media, contraindication for magnetic resonance imaging, pre-interventionally proven carcinoma of the prostate, and renal failure (glomerular filtration rate $<60 \mathrm{~mL} / \mathrm{min}$ ).

\section{Interventions}

PAE was performed by an experienced interventional radiologist (LH) who was familiar with the procedure. A $16 \mathrm{~F}$ transurethral catheter was inserted before surgery. A unilateral femoral sheath was placed in the right common femoral artery under local anaesthesia. The prostatic arterial supply was identified by selective internal iliac arteriography by a $5 \mathrm{~F}$ angiocatheter (Merit Medical). Prostatic arteries were selectively catheterised with 1.9-2.4 F microcatheters (Parkway soft; Asahi Intecc; Progreat; Terumo; Direxion; Boston Scientific) and embolised by use of 250-400 $\mu \mathrm{m}$ Embozene microspheres (Boston Scientific). Cone beam computed tomography was applied to identify prostatic arteries or prevent off-target embolisation in patients with challenging anatomical conditions. ${ }^{20}$ PAE was performed according to established techniques, bilaterally if possible, ${ }^{21-23}$ and was considered successful in the absence of the normal blush of the prostate and complete stasis of flow in the prostate arteries on angiography after embolisation. The transurethral catheter was removed the morning after the intervention.

Monopolar TURP was performed under spinal or general anaesthesia by an experienced study physician (DA, H-PS, DSE, or LM) using a $24 \mathrm{~F}$ resectoscope (Karl Storz Endoskope) with a cutting power of $180 \mathrm{~W}$ and a coagulation power of $60 \mathrm{~W}$, a standard tungsten wire 
loop, and electrolyte free mannitol-sorbitol solution (Purisole, Fresenius Kabi AG). Technical conduct of TURP adhered to generally accepted standards described elsewhere, ${ }^{24}$ and was performed step by step for the middle lobe, lateral lobes, ventral part, and apical residual tissue with the surgical capsule of the prostate serving as a landmark. A $20 \mathrm{~F}$ three way catheter was inserted for irrigation after resection, and left for at least two days depending on postoperative haematuria.

All patients received perioperative antibiotic prophylaxis, which was discontinued after removal of the bladder catheter or after three days at the latest (ciprofloxacin $500 \mathrm{mg}$ twice daily). An antiinflammatory (diclofenac $75 \mathrm{mg}$ twice daily) and acid suppressant (pantoprazole $40 \mathrm{mg}$ once daily) were administered for one week. Prostatic drug treatments were discontinued on the day of TURP and two weeks after PAE (owing to supposed slower efficacy).

\section{Outcomes}

Data were collected before intervention (baseline), during participants' stay in hospital, and at three scheduled follow-up visits at one, six, and 12 weeks after surgery. The primary outcome was the change from baseline to 12 weeks in the self administered IPSS questionnaire score, with scores ranging from 0 to 35 points ( $\leq 7$ : mild symptoms, 8 -19: moderate symptoms, $\geq 20$ points: severe symptoms). ${ }^{25}$ Secondary endpoints were assessed at scheduled visits and comprised:

- $\quad$ Free uroflowmetry

- Postvoid residual urine assessed by transabdominal ultrasound

- Quality of life related to lower urinary tract symptoms (ranging from 0 (“delighted") to 6 (“terrible"))

- Questionnaire chronic prostatitis symptoms index (CPSI) that assesses pain, voiding, and quality of life (score ranging from 0 (best) to 43 (worst))

- International index of erectile function short form 5 (IIEF) (score ranging from 0 (worst) to 25 (best))

- $\quad$ Blood tests for haemoglobin and prostate specific antigen

- $\quad$ Bladder diaries

- Assessment of adverse events according to the modified Clavien system ${ }^{26}$ and common terminology criteria for adverse events. ${ }^{27}$

Prostate volume assessment using magnetic resonanceimaging (BiopSee 2.2, Medcom) and pressure flow studies according to the standards recommended by the International Continence Society ${ }^{28}$ were performed at the baseline and 12 week visits. During the participants' stay in hospital, postoperative pain was assessed on a visual analogue scale (ranging from 0 (no pain) to 10 (maximum pain)). Assessment of procedural parameters included operation time, weight of resected tissue (for TURP), success of embolisation (monolateral or bilateral), and radiation dose (for PAE). Questionnaires were completed in the absence of the physician before visits. All patients with indwelling bladder catheters at baseline were asked to complete baseline questionnaires on the basis of their situation before catheter insertion (longest bladder catheter indwelling time before IPSS assessment was two weeks).

Additional secondary outcomes included changes in inflammatory blood parameters (eg, cytokines) and magnetic resonance imaging based analyses of tissue vascularisation after PAE. These secondary outcomes will be analysed in separate publications.

\section{Sample size and statistical methods}

Data analysis was performed per protocol.The study was designed as a non-inferiority trial with respect to the primary endpoint: the change in IPSS from baseline to 12 weeks. A change in IPSS of 3.0 points has previously been shown to be perceived by patients as a "slight improvement" only. Therefore, we considered a difference in mean IPSS change between both groups smaller than 3 points as showing non-inferiority for PAE. ${ }^{25}$ Based on previous findings, ${ }^{29}$ a standard deviation of 4.6 was expected for the change in IPSS. Thus, if the true mean difference was 0 , a one sided $t$ test with a significance level of 0.025 would have $80 \%$ power to reject the null hypothesis that PAE was inferior to TURP, with a sample size of 38 patients per group. Assuming a dropout rate of $20 \%$ during long term follow-up, we therefore aimed to include 100 patients.

The change in IPSS between baseline and 12 weeks was compared between groups by a one sided $t$ test for the non-inferiority of PAE, with a significance level of 0.025 . An additional analysis of the primary outcome was adjusted for IPSS at baseline by use of analysis of covariance. Secondary endpoints were treated as standard superiority comparisons, and means and 95\% confidence intervals at baseline and each follow-up visit are reported. We compared changes from baseline to follow-up visits between treatment groups with two sided $t$ tests for non-equality of means. All reported statistics were calculated from the available data. Thus, occasional missing values for some secondary outcomes cause the mean change from baseline to a follow-up visit (reported in text) to differ slightly from the difference between mean values at individual time points (reported in figures).

Mann-Whitney $U$ tests were used instead of $t$ tests for ordinal endpoints with less than 10 levels and for numerical endpoints that were clearly not normally distributed. We compared the overall frequency of adverse events in the two treatment groups to frequencies expected from patient numbers in the two groups with an exact binomial test. Relative frequencies of particular safety outcomes were compared between treatment groups with two sided tests for non-equality of proportions.

\section{Randomisation}

We performed randomisation using the data management software SecuTrial, stratifying for patient age ( $<70$ or $\geq 70$ years) and prostate volume $(<50$ or $\geq 50$ $\mathrm{mL}$ ) through minimisation. SecuTrial was programmed by the clinical trials unit's data manager, and automatic 
treatment allocation by SecuTrial was determined for individual patients without a predefined sequence after inclusion and entry of baseline characteristics by the investigators.

\section{Patient involvement}

No patients were involved in setting the research question, trial design, outcome measures, or recruitment of the study. Patients will be informed by press reports and the hospital's homepage following this publication.

\section{Results}

Study patients

The distribution of patients at screening, randomisation, treatment, and follow-up is shown in the CONSORT flowchart (fig 1). Of 144 patients assessed for eligibility, 41 were excluded because they did not meet inclusion criteria $(n=22)$, declined to participate $(n=15)$, or for other reasons $(n=4)$. Thus, 103 patients were randomly assigned to PAE or TURP between 11 February 2014 and 24 May 2017. Two patients allocated to PAE and one allocated to TURP refused to undergo surgery after randomisation and were excluded from the study. PAE was not possible due to vascular disorders in one patient, who underwent laser vapourisation of the prostate subsequently and was also excluded from the analysis. All 48 patients receiving PAE and 51 patients receiving TURP were available for the 12 week follow-up visit that included the primary outcome assessment. None needed an indwelling bladder catheter at this time. Patient characteristics and demographic data are provided in table 1.

\section{Primary endpoint}

Both treatments improved BPH-LUTS similarly. The mean change in IPSS from baseline to 12 weeks was -9.23 points after PAE and -10.77 points after TURP,

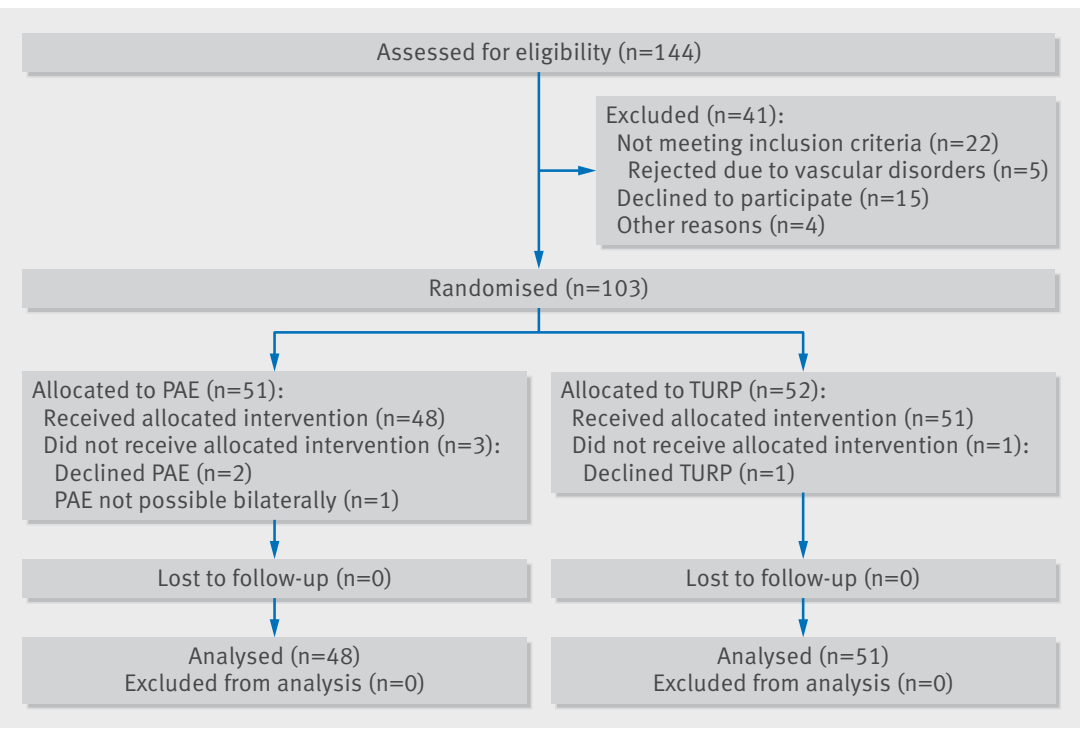

Fig 1 | Study enrolment and randomisation (CONSORT flow diagram) and the difference of 1.54 points in favour of TURP was not significant $(\mathrm{P}=0.31$; fig $2 \mathrm{~A})$. Non-inferiority of PAE (difference between means $<3$ points) could still not be established, owing to the large variation among individual outcomes ( $95 \%$ confidence interval for mean difference -1.45 to 4.52 points, $\mathrm{P}=0.17$ for test for non-inferiority; fig 2B).

A baseline adjusted analysis of covariance of the primary endpoint showed a mean difference between treatments of 2.87 points in favour of TURP (95\% confidence interval 0.53 to $5.21 ; \mathrm{P}=0.46$ for test for non-inferiority of PAE).

\section{Secondary endpoints}

Perioperative data and recovery parameters are shown in table 2. Compared with PAE, procedural time was statistically significantly shorter for TURP, but PAE showed favourable results in terms of blood loss, bladder catheter indwelling time, and duration of hospital stay.

In accordance with the IPSS, changes from baseline to 12 weeks did not differ statistically significantly for all other patient reported outcomes after PAE and TURP. IPSS related quality of life improved by -2.33 points after PAE and -2.69 points after TURP (difference 0.35 in favour of TURP (95\% confidence interval -0.30 to 1.00 ); $\mathrm{P}=0.15$; fig $2 \mathrm{C}$ ). The chronic prostatitis symptoms index improved by -7.83 points versus -7.16 points (difference 0.67 in favour of PAE ( -3.65 to -2.32$)$; $\mathrm{P}=0.66$; fig $2 \mathrm{D})$. The international index of erectile function changed by -0.98 points versus -1.84 points (difference 0.87 in favour of PAE (-1.89 to 3.63); $\mathrm{P}=0.53$; fig $2 \mathrm{E}$ ).

By contrast, functional outcomes after 12 weeks were clearly in favour of TURP. After PAE and TURP, we saw an improvement in maximum rates of free urinary flow by $5.19 \mathrm{~mL} / \mathrm{s}$ versus $15.34 \mathrm{~mL} / \mathrm{s}$ (difference 10.15 in favour of TURP (95\% confidence interval -14.67 to -5.63$)$; $\mathrm{P}<0.001$; fig $3 \mathrm{~A}$ ) and changes in postvoid residual urine by $-86.36 \mathrm{~mL}$ versus $-199.98 \mathrm{~mL}$ (difference 113.62 in favour of TURP (39.25 to 187.98); $\mathrm{P}=0.003$; fig 3B). A marked initial increase in prostate specific antigen after PAE largely vanished after 12 weeks, leading to reductions by $2.00 \mu \mathrm{g} / \mathrm{L}$ versus $3.11 \mu \mathrm{g} / \mathrm{L}$ (difference 1.11 in favour of TURP $(-0.89$ to 3.10); $\mathrm{P}=0.07$; fig $3 \mathrm{C}$ ). The mean change in prostate volume as measured by magnetic resonance imaging volumetry from baseline to 12 weeks after PAE and TURP was $-12.17 \mathrm{~mL}$ versus $-30.27 \mathrm{~mL}$ (difference 18.11 in favour of TURP (10.11 to 26.10); $\mathrm{P}<0.001$; fig 3D). Pressure flow studies showed significant disadvantages for PAE compared with TURP for the reduction of bladder outlet obstruction, indicated by a change in detrusor pressure at maximum urinary flow rate of $-17.17 \mathrm{~cm} \mathrm{H}_{2} \mathrm{O}$ versus $-41.07 \mathrm{~cm} \mathrm{H}_{2} \mathrm{O}$ (difference 23.91 in favour of TURP (9.09 to 38.72); $\mathrm{P}=0.002$; fig $3 \mathrm{E}$ ) and a shift towards a less obstructive category in $56 \%$ of patients after PAE and $93 \%$ after TURP (proportion test, $\mathrm{P}=0.003$; fig $3 \mathrm{~F}$ ).

Bladder diaries showed functional improvements from baseline to 12 weeks without significant 


\begin{tabular}{|c|c|c|}
\hline Characteristic & $\operatorname{PAE}(n=48)$ & TURP $(n=51)$ \\
\hline Age (years) & $65.7(9.3)$ & $66.1(9.8)$ \\
\hline Body mass indext & $26.5(4.2)$ & $27.0(3.9)$ \\
\hline Charlson comorbidity index & $3.6(1.6)$ & $4.3(2.1)$ \\
\hline IPSS & $19.38(6.37)$ & $17.59(6.17)$ \\
\hline Prostate specific antigen concentration $(\mu \mathrm{g} / \mathrm{L})$ & $4.2(5.4)$ & $4.5(5.6)$ \\
\hline \multicolumn{3}{|l|}{ Prostate volume $(\mathrm{mL})$} \\
\hline Measured by transabdominal ultrasound & $51.2(16.5)$ & $52.1(18.6)$ \\
\hline Measured by magnetic resonance imaging & $52.8(32.0)$ & $56.5(31.1)$ \\
\hline \multicolumn{3}{|c|}{ Medical treatment of lower urinary tract symptoms before surgery (No (\%) of patients) } \\
\hline 5a-reductase inhibitors & 0 & $1(2.0)$ \\
\hline a1-adrenergic receptor antagonists & $26(54.2)$ & $22(43.1)$ \\
\hline Combination of both drugs mentioned above & $14(29.2)$ & $13(25.5)$ \\
\hline Antimuscarinic drugs & $8(16.7)$ & $9(17.6)$ \\
\hline Total No receiving drug treatment & $41(85.4)$ & $44(86.3)$ \\
\hline Indwelling urethral catheter at baseline (No (\%) of patients) & $9(18.8)$ & $12(24.5)$ \\
\hline
\end{tabular}

differences between PAE and TURP. Mean voided volume increased by $28.7 \mathrm{~mL}$ versus $18.9 \mathrm{~mL}$ (difference 9.8 in favour of PAE ( $95 \%$ confidence interval -38.94 to 58.54); $\mathrm{P}=0.69$ ), daytime frequency of urination decreased by 0.75 versus 0.89 (difference 0.14 in favour of TURP ( -1.08 to 1.37$) ; \mathrm{P}=0.81$ ), and nocturia decreased by 0.35 versus 0.21 (difference 0.14 in favour of PAE ( -0.83 to 0.55$) ; \mathrm{P}=0.68)$.

\section{Safety}

Figure 4 shows the frequency of particular types of adverse events. Treatment related adverse events were half as frequent after PAE than after TURP during the first 12 weeks of the study $(\mathrm{n}=36 v \mathrm{n}=70 ; \mathrm{P}=0.003$; fig 4). The risk of at least one treatment related adverse event did not differ significantly between groups $(62.5 \% \vee 70.6 \%$, relative risk after PAE 0.89 (95\% confidence interval 0.67 to 1.17$), \mathrm{P}=0.52$ ), but fewer patients had two or more treatment related adverse events after PAE than after TURP $(16.7 \% v 45.1 \%$, 0.37 (0.18 to 0.75$), \mathrm{P}=0.005)$. The distribution of adverse events among severity classes ${ }^{26}$ was similar for both treatments $(\mathrm{P}=0.44)$. Clavien grades 1,2 , and 3 were assigned to $71.4 \%, 22.9 \%$, and $5.7 \%$ of the adverse events after PAE, respectively and to $64.5 \%$, $24.2 \%$, and $11.3 \%$ of the adverse events after TURP, respectively.

Postoperative pain was reported by more patients after PAE than after TURP $(56.3 \% v 31.9 \%$, relative risk 1.76 (95\% confidence interval 1.08 to 2.87 ); $\mathrm{P}=0.03$ ). Among patients who reported pain, the maximum pain intensity tended to be higher after PAE than after TURP (mean score on visual analogue scale $4.0 v 3.1$ ), but the difference $(0.9$ points ( $95 \%$ confidence interval 0.76 to $2.42)$ ) was not significant $(P=0.28)$. Severe pain $(\geq 6$ points on visual analogue scale) was experienced by nine $(18.8 \%)$ patients after PAE and two $(4.3 \%)$ after TURP (relative risk 4.41 (95\% confidence interval 1.00 to 19.33); $\mathrm{P}=0.06$ ), but was limited to the first $12-24$ hours after the procedure.

Complications specific to angiointerventions (that is, endovascular interventions) were limited to one patient with moderate haematoma at the puncture site, one with postembolisation syndrome including fever (lasting <24 h), and one with ischaemia in a limited area of the bladder wall requiring transurethral removal of necrotic tissue. One patient reported de novo erectile dysfunction despite the use of highly selective embolisation, and 56\% (14/25) of patients in whom ejaculation was assessable had ejaculatory dysfunction after PAE compared with 84\% (21/25) after TURP (relative risk 0.67 (95\% confidence interval 0.45 to 0.98$) ; \mathrm{P}=0.06)$.

\section{Discussion}

\section{Principal findings}

This randomised trial provides reliable comparative data that enhance the so far inconsistent and low quality evidence available for $\mathrm{PAE},{ }^{11}$ and outlines its advantages and disadvantages compared with TURP. Our results indicate that similar degrees of improvement in symptoms are achieved with PAE and TURP in the short term, although PAE has slightly smaller improvements than TURP. However, clear advantages of TURP over PAE were found regarding the pure desobstructive effect, reflected in improved micturition parameters and a reduction in bladder outlet obstruction.

Surgery for benign prostatic hyperplasia is usually performed for refractory symptoms associated with reduced quality of life; thus, patient reported outcomes are generally considered the most representative primary outcome. ${ }^{5}$ In this regard, both TURP and PAE have yielded effect sizes in the improvement of BPHLUTS defined as "marked" elsewhere. ${ }^{25}$ Nevertheless, TURP has a more pronounced, pure urodynamic 

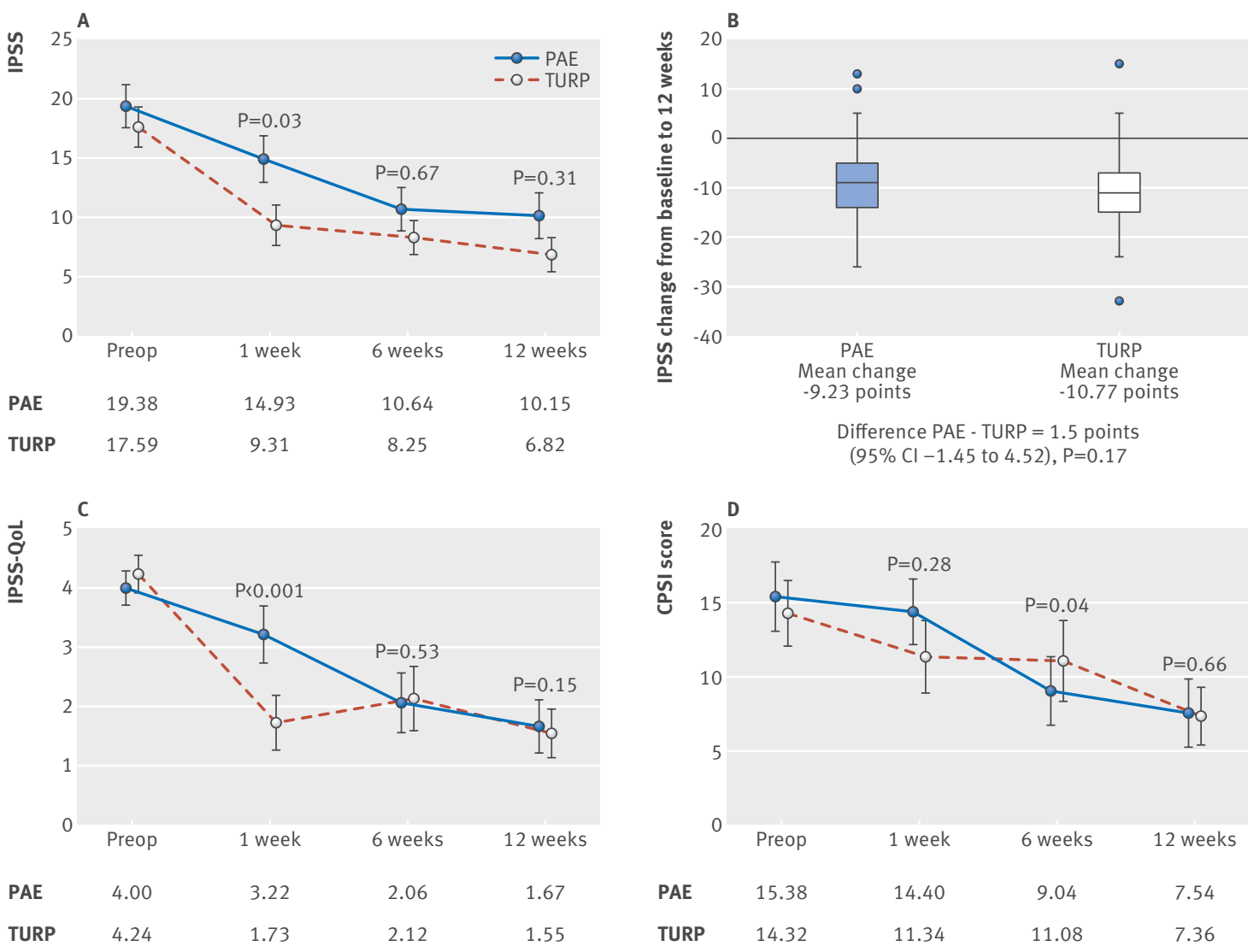

$\begin{array}{lllll}\text { PAE } & 4.00 & 3.22 & 2.06 & 1.67 \\ \text { TURP } & 4.24 & 1.73 & 2.12 & 1.55\end{array}$

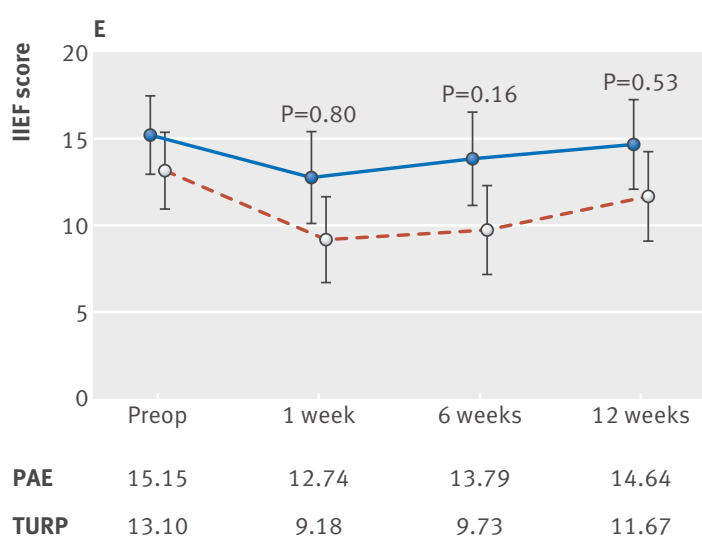

Fig 2 | Primary outcome and patient reported secondary outcomes in patients with lower urinary tract symptoms secondary to benign prostatic hyperplasia receiving prostatic artery embolisation (PAE) or transurethral resection of the prostate (TURP). (A) Improvements in the international prostate symptom score (IPSS) after PAE and TURP. (B) Mean difference in IPSS (primary efficacy endpoint) between treatment groups. Secondary endpoints between PAE and TURP for (C) IPSS related quality of life (QoL); (D) chronic prostatitis symptoms index (CPSI) assessing pain, urinary symptoms, and quality of life; and (E) international index of erectile function (IIEF). I bars in (A, C, D, E) indicate $95 \%$ confidence intervals. Boxes in (B) show the interquartile range, with central lines indicating the median. Each whisker extends to the most extreme data point, which deviates no more than 1.5 times the interquartile range from the box. Points indicate observations, which lie beyond the extremes of the whiskers. Preop=before the operation (that is, baseline). $P$ values (apart from part B) are reported for differences of change from baseline between both treatments. Means are calculated from the complete dataset at each visit. Occasional missing values for parts (D) and (E) cause slight differences between the mean change from baseline to a follow-up visit and the difference between mean values at individual time points reported in the text

desobstructive effect than PAE. Thus, TURP should be preferred in patients with complications associated with bladder outlet obstruction, such as repeated acute urinary retention, bladder diverticula or stones, impaired renal function due to bladder outlet obstruction, and weak detrusor pressure combined with high amounts of postvoid residual urine (although some of these patients were excluded from our study). 


\begin{tabular}{|c|c|c|c|}
\hline Measure & $\operatorname{PAE}(n=48)$ & TURP $(n=51)$ & $\mathbf{P}$ \\
\hline \multicolumn{4}{|l|}{ Anaesthesia } \\
\hline General (No (\%) of patients) & - & $26(51)$ & - \\
\hline Spinal (No (\%) of patients) & - & $25(49)$ & - \\
\hline Local (No (\%) of patients) & $48(100)$ & - & - \\
\hline Procedure time (min) & $122.2(25.8)$ & $69.5(22.5)$ & $<0.001$ \\
\hline \multicolumn{4}{|l|}{ PAE procedure details } \\
\hline Bilateral (No (\%) of patients) & $36(75.0)$ & - & - \\
\hline Unilateral (No (\%) of patients) & $12(25.0)$ & - & - \\
\hline Fluoroscopy time (min) & $50.8(17.5)$ & - & - \\
\hline Dose area product $\left(\mathrm{Gy} / \mathrm{cm}^{2}\right)$ & $176.5(101.2)$ & - & - \\
\hline Pain during intervention (visual analogue scale) & $0.1(0.6)$ & - & - \\
\hline Additional analgesics needed (No (\%) of patients)* & $2(4.2)$ & - & - \\
\hline Amount of embolisation particles used $(\mathrm{mL})$ & $1.0(0.4)$ & - & - \\
\hline \multicolumn{4}{|l|}{ TURP procedure details } \\
\hline Time of resection (min) & - & $58.25(24.33)$ & - \\
\hline Weight of resected tissue (g) & - & $25.20(15.16)$ & - \\
\hline Retrieval rate (g/min) & - & $0.43(0.20)$ & - \\
\hline \multicolumn{4}{|l|}{ Recovery parameters } \\
\hline Preoperative haemoglobin (g/L) & $147.4(12.2)$ & $145.2(12.7)$ & 0.38 \\
\hline Haemoglobin decrease $24 \mathrm{~h}(\mathrm{~g} / \mathrm{L})$ & $-4.3(7.0)$ & $-13.8(11.0)$ & 0.001 \\
\hline Bladder catheter indwelling time (days) & $1.3(1.4)$ & $3.3(1.4)$ & 0.001 \\
\hline Duration of hospital stay (days) & $2.2(0.6)$ & $4.2(1.7)$ & 0.001 \\
\hline
\end{tabular}

The apparent contradiction between patient reported and functional outcomes can be explained by the poor correlation between urodynamic obstruction and subjective perception of symptoms described previously, ${ }^{30}$ and the fact that storage symptomswhich improve less than voiding symptoms after conventional surgery-are usually more bothersome to patients. ${ }^{30-32}$ Similar findings have been reported for other minimally invasive procedures such as transurethral needle ablation or microwave thermotherapy of the prostate and were associated with higher reintervention rates. ${ }^{33} 34$ In our study, considerably fewer and less severe adverse events were found after PAE, which could be performed under local anaesthesia and was associated with reduced blood loss and shorter duration of hospital stay and catheterisation than TURP.

\section{Strengths and weaknesses of the study}

Strengths of the study included its interdisciplinary study setting and the independent trial monitoring and data management. The study population and outcomes after TURP were similar to large series of real world data on TURP published previously and our results seem to represent clinical routine. ${ }^{7}$ Moreover, the study followed up all patients treated according to randomisation, and provided a detailed investigation of adverse events.

Our study has some limitations. The number of patients was not high enough to conclusively determine non-inferiority or inferiority of PAE versus TURP, despite the difference between the two procedures being less than the defined threshold of 3 IPSS points. Non-inferiority could not be demonstrated conclusively in the primary analysis because variability of the change in IPSS among patients in both groups (standard deviation 7.5) was larger than the value of 4.6 assumed in the sample size calculation. A main cause for this discrepancy might have been different inclusion criteria and patient characteristics in the study taken as a basis. ${ }^{29}$ In addition, the difference between PAE and TURP in the additional baseline adjusted analysis was only slightly below 3 points (that is, 2.87; $\mathrm{P}=0.46$ for non-inferiority), which further outlines a need for larger scale trials.

With our study design, treatment groups might not have been perfectly balanced with respect to prognostic factors other than the two stratifying factors-for example, the degree of obstruction or comorbidities. However, separate analyses with adjustment for all these factors showed that they were unrelated to our primary outcome (change in IPSS) and did not influence test results for differences between PAE and TURP.

Blinding of patients and physicians was not feasible in the framework of our trial. Therefore, both patients and physicians might have been biased in favour of or against a new treatment. Expert bias cannot be excluded, because one interventional radiologist performed all embolisations. The evaluation of patient satisfaction during mid-term and long term follow-up will be crucial because of a potential placebo effect, the fact that TURP might be more bothersome than PAE in the first 12 weeks, and the possibility of a further treatment effect beyond 12 weeks after PAE. Moreover, the significant difference between both treatments with regard to desobstruction might lead to higher reintervention rates after PAE over the long term.

\section{Strengths and weaknesses in relation to other studies}

Evidence regarding the efficacy and safety of PAE in the treatment of BPH-LUTS has mainly been based on proofof-concept trials and cohort studies so far, and the three published trials providing comparative data have had substantial methodological limitations. ${ }^{11}$ While Russo and colleagues compared PAE with open prostatectomy in a matched pair non-randomised study, ${ }^{14}$ Carnevale and colleagues ${ }^{15}$ randomised only 30 patients to PAE or TURP. The trial ${ }^{15}$ was not registered, and did not report on a clearly defined primary outcome measure. The largest randomised controlled study published so far by Gao and colleagues ${ }^{13}$ has been questioned by the opinion leaders in the field of PAE because of major inconsistencies, including a limited adherence to fundamental standards of scientific practice, underreporting of adverse events, and an "over-reporting” of the results of PAE. ${ }^{35}$

So far, little has been reported on PAE associated complications, and a higher complication rate for PAE than for TURP in pooled analyses of comparative trials was attributed to substantial under-reporting of complications after TURP. ${ }^{811}$ Our results elucidate 

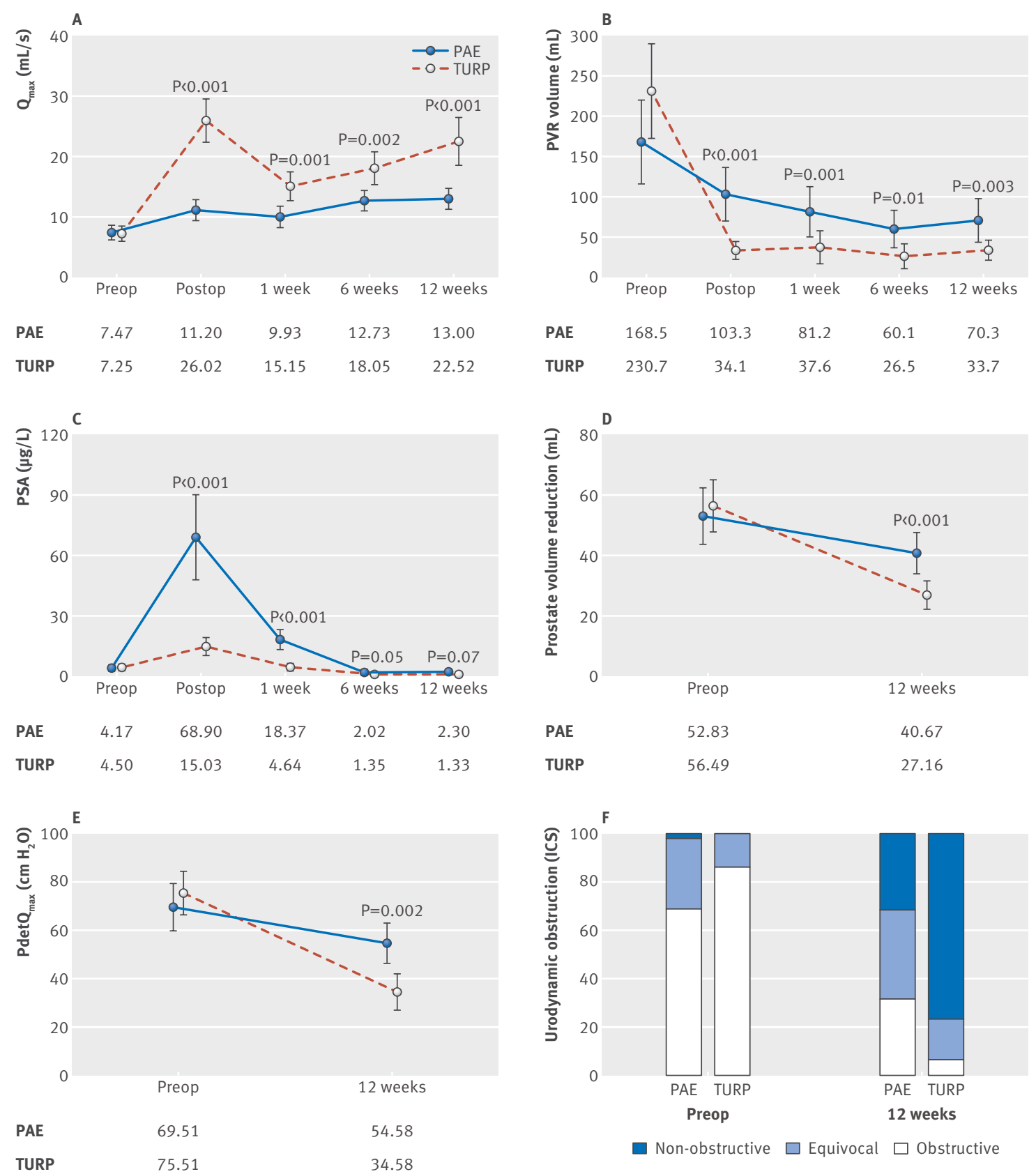

Fig 3 | Functional secondary outcome parameters in patients with lower urinary tract symptoms secondary to benign prostatic hyperplasia receiving prostatic artery embolisation (PAE) or transurethral resection of the prostate (TURP). Parameters include (A) maximum urinary flow rate, (B) postvoid residual urine, (C) concentrations of prostate specific antigen (PSA), (D) Prostate volume reduction as assessed by magnetic resonance imaging, (E) desobstruction as measured by detrusor pressure at maximum flow rate (PdetQmax), and (F) urodynamic obstruction as measured by International Continence Society (ICS) classification. I bars $=95 \%$ confidence intervals; dots=means calculated from the complete dataset at each visit. $P$ values are reported for differences of change from baseline between both treatments. Preop=before the operation (that is, baseline); postop=after the operation. Occasional missing values for secondary outcomes may cause the mean change from baseline to a follow-up visit in (parts A-E) to differ slightly from the difference between mean values at individual time points reported in the text

these issues and illustrate that the scope of adverse events after PAE-although favourable compared to TURP-has also been underestimated so far. Preservation of ejaculatory function is a fundamental aspect for many patients and has to be discussed before any treatment is initiated. Ejaculatory disorders have so far been reported in only two of more than
1000 cases in the literature, ${ }^{11}$ but were encountered in our series in $56 \%$ of patients receiving PAE; this finding was unexpected and needs clarification in the future.

Althoughduration of hospitalstayand catheterisation are practice dependent and were handled rather conservatively after TURP in this trial, PAE has been 


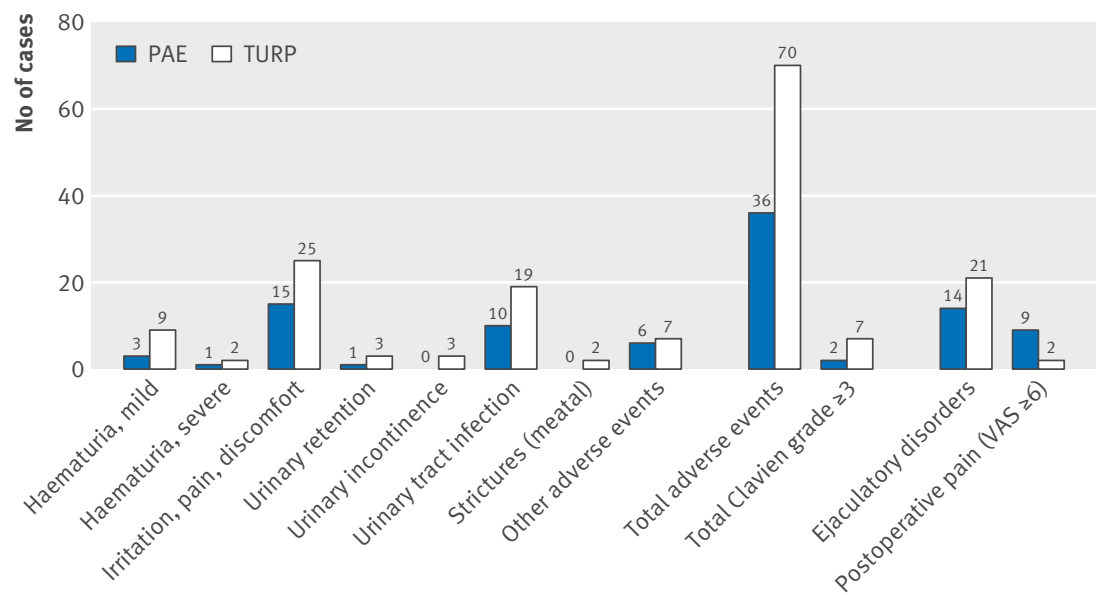

Fig 4 | Frequency of treatment related adverse events, postoperative pain, and ejaculatory disorders in patients with lower urinary tract symptoms secondary to benign prostatic hyperplasia receiving prostatic artery embolisation (PAE) or transurethral resection of the prostate (TURP). VAS=visual analogue scale $(\geq 6$ points=severe pain). Postoperative pain during hospital stay and ejaculatory disorders were not defined as deviation from the normal postoperative cours $\mathrm{e}^{26}$; therefore, these events were not included in the total number of adverse events but assessed separately

shown to be feasible even without catheterisation and in an outpatient setting. ${ }^{36}$ Radiation exposure of the patients receiving PAE was clearly below the thresholds recommended for such interventions by the Swiss Federal Office of Public Health ${ }^{37}$ and the mean total dose area product in this study was less than half than in other studies, ${ }^{38}$ indicating that the potential to improve radiation protection during PAE is currently clearly not yet fully exploited.

Follow-up in the trials mentioned above was for up to two years. Although assessment in our trial will continue for up to five years, the primary outcome was deliberately assigned to the visit after 12 weeks, because patients expect an outcome of a surgical intervention at this time.

\section{Meaning of the study findings, unanswered questions, and future research}

The findings of this study elucidate the efficacy and safety profile of PAE. Our findings provide valuable information regarding patient selection and counselling, and will be useful for designing further trials in this field.

Larger scale trials and long term follow-up data are needed before PAE is implemented as a routine treatment for BPH-LUTS. The large standard deviation in our trial emphasises that such trials should also include subgroup analyses to detect patients who benefit most from PAE, which was beyond the scope of our study. Future research should also focus on the technical aspects of PAE, to determine the most efficient and safe approach with this intervention. Further refinement of embolisation techniques might eventually enable an even greater treatment effect. Owing to its favourable side effect profile, trials comparing PAE with non-invasive treatments (such as $5 \alpha$-reductase inhibitors or combined treatments of $\alpha$-blockers and $5 \alpha$-reductase inhibitors) seem to be reasonable.

\section{Conclusions}

This study shows that PAE is a valuable alternative to TURP in the treatment of BPH-LUTS. PAE should be considered in patients in whom the indication for surgery is primarily based on symptoms. For severe complications related to benign prostatic hyperplasia, TURP should be preferred. Further comparative study findings including longer follow-up periods and subgroup analyses should be evaluated before PAE can be considered as a routine treatment.

Acknowledgements: This study included many important patient reported outcomes and we thank the trial participants for their great efforts and excellent adherence to the study protocol. We also thank Sarah Haile and Rafael Sauter for providing statistical advice during the planning phase of the study; the clinical trials unit of St Gallen Cantonal Hospital for their support in study coordination, data management, and trial monitoring; and Alistair Reeves for medical writing support.

Contributors: DA and LH did the literature search, designed the study, collected the data, interpreted the data, and wrote the report. DA is guarantor. GM collected the data, interpreted the data, and wrote the report. SM collected the data, and interpreted the data. SG did the statistical analysis, interpreted the data, and wrote the report. TMK, $\mathrm{H}-\mathrm{PS}$, and DSE designed the study, collected the data, interpreted the data, and wrote the report. LM did the literature search, designed the study, collected the data, interpreted the data, and wrote the report. All authors had full access to all of the data (including statistical reports and tables) in the study and can take responsibility for the integrity of the data and the accuracy of the data analysis. The corresponding author attests that all listed authors meet authorship criteria and that no others meeting the criteria have been omitted.

Funding: The trial was supported by a grant from the research committee of St Gallen Cantonal Hospital (14/08). The funder had no role in the conduct or analysis of the trial.

Competing interests: All authors have completed the ICMJE uniform disclosure form at www.icmje.org/coi_disclosure.pdf and declare: support from St Gallen Cantonal Hospital for the submitted work; no financial relationship with any organisations that might have an interest in the submitted work in the previous three years; no other relationships or activities that could appear to have influenced the submitted work.

Ethical approval: The study was approved by the local ethics committee (EKSG14/004), and all participants gave written informed consent before taking part in the study.

Data sharing: Participants gave consent for anonymised data sharing. Requests for an anonymised, full dataset of physician level data and statistical code will be considered if the proposed use aligns with public good purposes, does not conflict with other requests, does not conflict with the planned use by the trial steering committee, contingent on approval from the local ethics committee (EKOS). Requests can be addressed to the corresponding author.

The lead author affirms that the manuscript is an honest, accurate, and transparent account of the study being reported; that no important aspects of the study have been omitted; and that any discrepancies from the study as planned have been explained.

This is an Open Access article distributed in accordance with the Creative Commons Attribution Non Commercial (CC BY-NC 4.0) license, which permits others to distribute, remix, adapt, build upon this work non-commercially, and license their derivative works on different terms, provided the original work is properly cited and the use is noncommercial. See: http://creativecommons.org/licenses/by-nc/4.0/.

Berry SJ, Coffey DS, Walsh PC, Ewing LL. The development of human benign prostatic hyperplasia with age. J Urol 1984;132:474-9. doi:10.1016/S0022-5347(17)49698-4

2 Taub DA, Wei JT. The economics of benign prostatic hyperplasia and lower urinary tract symptoms in the United States. Curr Urol Rep 2006;7:272-81. doi:10.1007/s11934-996-0006-0 
3 van Exel NJ, Koopmanschap MA, McDonnell J, Chapple CR, Berges R, Rutten FF, TRIUMPH Pan-European Expert Panel. Medical consumption and costs during a one-year follow-up of patients with LUTS suggestive of BPH in six european countries: report of the TRIUMPH study. Eur Urol 2006;49:92-102. doi:10.1016/j. eururo.2005.09.016.

4 Malaeb BS, Yu X, McBean AM, Elliott SP. National trends in surgical therapy for benign prostatic hyperplasia in the United States (2000-2008). Urology 2012;79:1111-6. doi:10.1016/j. urology.2011.11.084

5 Gravas S, Cornu JN, Drake MJ, et al. EAU Guidelines on management of non-neurogenic male lower urinary tract symptoms (LUTS), incl. benign prostatic obstruction (BPO). 2018 https://uroweb.org/ guideline/treatment-of-non-neurogenic-male-luts/.

6 McVary KT, Roehrborn CG, Avins AL, et al. American Urological Association Guideline: management of benign prostatic hyperplasia (BPH). Revised, 2010. https://www.auanet.org/benign-prostatichyperplasia-(2010-reviewed-and-validity-confirmed-2014).

7 Reich O, Gratzke C, Bachmann A, et al, Urology Section of the Bavarian Working Group for Quality Assurance. Morbidity, mortality and early outcome of transurethral resection of the prostate: a prospective multicenter evaluation of 10,654 patients. J Urol 2008:180:246-9. doi:10.1016/j.juro.2008.03.058.

8 Ahyai SA, Gilling P, Kaplan SA, et al. Meta-analysis of functional outcomes and complications following transurethral procedures for lower urinary tract symptoms resulting from benign prostatic enlargement. Eur Urol 2010;58:384-97. doi:10.1016/j. eururo.2010.06.005.

9 Lukacs B, Cornu IN, Aout M, et al. Management of lower urinary tract symptoms related to benign prostatic hyperplasia in real-life practice in france: a comprehensive population study. Eur Urol 2013;64:493 501. doi:10.1016/j.eururo.2013.02.026.

10 DeMeritt JS, Elmasri FF, Esposito MP, Rosenberg GS. Relief of benign prostatic hyperplasia-related bladder outlet obstruction after transarterial polyvinyl alcohol prostate embolization. J Vasc Interv Radiol 2000;11:767-70. doi:10.1016/S10510443(07)61638-8

11 Shim SR, Kanhai KJ, Ko YM, Kim JH. Efficacy and safety of prostatic arterial embolization: systematic review with meta-analysis and meta-regression. J Urol 2017;197:465-79. doi:10.1016/j. juro.2016.08.100

12 Bagla S, Smirniotopoulos J, Orlando J, Piechowiak R. Cost analysis of prostate artery embolization (PAE) and transurethral resection of the prostate (TURP) in the treatment of benign prostatic hyperplasia. Cardiovasc Intervent Radiol 2017;40:1694-7. doi:10.1007/s00270017-1700-7.

13 Gao YA, Huang Y, Zhang R, et al. Benign prostatic hyperplasia: prostatic arterial embolization versus transurethral resection of the prostate-a prospective, randomized, and controlled clinical trial. Radiology 2014;270:920-8. doi:10.1148/radiol.13122803.

14 Russo Gl, Kurbatov D, Sansalone S, et al. Prostatic arterial embolization vs open prostatectomy: a 1-year matched-pair analysis of functional outcomes and morbidities. Urology 2015;86:343-8. doi:10.1016/j.urology.2015.04.037.

15 Carnevale FC, Iscaife A, Yoshinaga EM, Moreira AM, Antunes AA, Sroug $M$. Transurethral resection of the prostate (TURP) versus original and perfected prostate artery embolization (PAE) due to benign prostatic hyperplasia (BPH): preliminary results of a single center, prospective, urodynamic-controlled analysis. Cardiovasc Intervent Radiol 2016;39:44-52. doi:10.1007/s00270-015-1202-4

16 McWilliams JP, Kuo MD, Rose SC, et al, Society of Interventional Radiology. Society of Interventional Radiology position statement: prostate artery embolization for treatment of benign disease of the prostate. J Vasc Interv Radiol 2014;25:1349-51. doi:10.1016/j. jvir.2014.05.005

17 World Medical Association. Declaration of Helsinki - ethical principles for medical research involving human subjects. 1964. https://www. wma.net/what-we-do/education/medical-ethics-manual/.

18 International conference on harmonisation: Good clinical practice guideline. http://www.ich.org/products/guidelines/efficacy/article/ efficacyguidelines. html.

19 Abt D, Mordasini L, Hechelhammer L, Kessler TM, Schmid HP, Engeler DS. Prostatic artery embolization versus conventional TUR-P in the treatment of benign prostatic hyperplasia: protocol for a prospective randomized non-inferiority trial. BMC Urol 2014;14:94. doi:10.1186/1471-2490-14-94.

20 Bagla S, Rholl KS, Sterling KM, et al. Utility of cone-beam CT imaging in prostatic artery embolization. J Vasc Interv Radiol 2013;24:1603 7. doi:10.1016/j.jvir.2013.06.024.
21 Martins Pisco J, Pereira J, Rio Tinto H, Fernandes L, Bilhim T. How to perform prostatic arterial embolization. Tech Vasc Interv Radiol 2012:15:286-9. doi:10.1053/i.tvir.2012.09.002

22 Carnevale FC, Antunes AA. Prostatic artery embolization for enlarged prostates due to benign prostatic hyperplasia. How I do it. Cardiovasc Intervent Radiol 2013;36:1452-63. doi:10.1007/s00270-0130680-5.

23 Carnevale FC, Moreira AM, Antunes AA. The "PErFecTED technique": proximal embolization first, then embolize distal for benign prostatic hyperplasia. Cardiovasc Intervent Radiol 2014;37:1602-5. doi:10.1007/s00270-014-0908-z.

24 Milam DF. Transurethral resection of the prostate. In: Smith JA, Howards SS, McGuire EJ, Preminger GM, eds. Hinman's atlas of urologic surgery. Elsevier, 2012. doi:10.1016/B978-1-4160-42105.00080-3.

25 Barry MJ, Williford WO, Chang Y, et al. Benign prostatic hyperplasia specific health status measures in clinical research: how much change in the American Urological Association symptom index and the benign prostatic hyperplasia impact index is perceptible to patients? J Urol 1995;154:1770-4. doi:10.1016/S00225347(01)66780-6

26 Dindo D, Demartines N, Clavien PA. Classification of surgical complications: a new proposal with evaluation in a cohort of 6336 patients and results of a survey. Ann Surg 2004;240:205-13. doi:10.1097/01.sla.0000133083.54934.ae

27 National Cancer Institute ( $\mathrm{NCl}$ ) common terminology criteria for adverse events. (CTCAE) version 4.0. https://evs.nci.nih. gov/ftp1/CTCAE/CTCAE_4.03/CTCAE_4.03_2010-06-14_ QuickReference $5 \times 7$.pdf.

28 Schäfer W, Abrams P, Liao L, et al, International Continence Society. Good urodynamic practices: uroflowmetry, filling cystometry, and pressure-flow studies. Neurourol Urodyn 2002;21:261-74. doi:10.1002/nau.10066

29 Neyer M, Reissigl A, Schwab C, et al. Bipolar versus monopolar transurethral resection of the prostate: results of a comparative, prospective bicenter study-perioperative outcome and long-term efficacy. Urol Int 2013;90:62-7. doi:10.1159/000343688.

30 Barry MJ, Cockett AT, Holtgrewe HL, McConnell JD, Sihelnik SA, Winfield HN. Relationship of symptoms of prostatism to commonly used physiological and anatomical measures of the severity of benign prostatic hyperplasia. J Urol 1993;150:351-8. doi:10.1016/ S0022-5347(17)35482-4

31 Hakenberg OW, Pinnock CB, Marshall VR. Does evaluation with the International Prostate Symptom Score predict the outcome of transurethral resection of the prostate? J Urol 1997;158:94-9. doi:10.1097/00005392-199707000-00025.

32 Peters TJ, Donovan JL, Kay HE, et al. The International Continence Society "benign prostatic hyperplasia" study: the botherosomeness of urinary symptoms. J Urol 1997;157:885-9. doi:10.1016/S00225347(01)65075-4

33 Hill B, Belville W, Bruskewitz R, et al. Transurethral needle ablation versus transurethral resection of the prostate for the treatment of symptomatic benign prostatic hyperplasia: 5-year results of a prospective, randomized, multicenter clinical trial. J Urol 2004;171:2336-40. doi:10.1097/01.ju.0000127761.87421. $\mathrm{aO}$

34 Mattiasson A, Wagrell L, Schelin S, et al. Five-year follow-up of feedback microwave thermotherapy versus TURP for clinical BPH: a prospective randomized multicenter study. Urology 2007;69:91-6, discussion 96-7. doi:10.1016/j.urology.2006.08.1115.

35 Bilhim T, Bagla S, Sapoval M, Carnevale FC, Salem R, Golzarian J. Prostatic arterial embolization versus transurethral resection of the prostate for benign prostatic hyperplasia. Radiology 2015;276:3101. doi:10.1148/radiol.2015141853.

36 Lebdai S, Delongchamps NB, Sapoval M, et al. Early results and complications of prostatic arterial embolization for benign prostatic hyperplasia. World J Urol 2016;34:625-32. doi:10.1007/s00345015-1665-6.

37 Swiss Federal Office of Public Health. Wegleitung R-06-05 Diagnostische Referenzwerte (DRW) für interventionelle radiologische Anwendungen (version 01.01.2018). https://www.bag. admin.ch/bag/de/home/themen/mensch-gesundheit/strahlungradioaktivitaet-schall/bewilligungen-aufsicht-im-strahlenschutz/ informationen-fuer-medizinische-betriebe/diagnostischereferenzwerte-im-strahlenschutz.html.

38 Andrade G, Khoury HJ, Garzón W], et al. Radiation exposure of patients and interventional radiologists during prostatic artery embolization: a prospective single-operator study. J Vasc Interv Radiol 2017;28:517-21. doi:10.1016/j.jvir.2017.01.005. 